\title{
In vitro assessment of some sperm function following exposure to levonorgestrel in human fallopian tubes
}

\author{
Alexia Hermanny ${ }^{1}$, M Valeria Bahamondes ${ }^{1}$, Francisco Fazano ${ }^{1}$, Nadia M Marchi', Maria Elena Ortiz², \\ Maria Heloisa RR Genghini ${ }^{1}$, Horacio B Croxatto ${ }^{3}$ and Luis Bahamondes ${ }^{1 *}$
}

\begin{abstract}
Background: The mechanism of action of levonorgestrel (LNG) as emergency contraception (EC) remains a subject of debate and its effect on sperm function has been only partially explained. The aim of this study was to assess whether LNG at a similar dose to those found in serum following oral intake for EC could affect spermatozoa when exposed to human fallopian tubes in vitro.

Methods: Fifteen mini-laparotomies were performed, the side on which ovulation occurred was recorded, and both tubes were removed and perfused with a suspension containing $1 \times 10(6)$ motile spermatozoa, with or without LNG. Following 4-hour incubation, the tubes were sectioned to separate the isthmus and the ampulla. Each segment was flushed and the material was evaluated to quantify the number of motile sperm, the number of spermatozoa adhering to the oviductal epithelium and the acrosome reaction (AR) rate.

Results: The addition of LNG did not significantly alter the number of recovered motile spermatozoa either at the isthmus or at the ampulla, nor did it have any effect on the number of recovered spermatozoa adhered to the human tubal epithelium. Furthermore, LNG did not affect the AR rate. No significant differences were found even when the side on which ovulation occurred was taken into account.

Conclusions: In a similar dose to that observed in serum following oral intake for EC, LNG had no effect on the number of motile spermatozoa recovered from the human fallopian tubes in vitro, on their adhesion to the tubal epithelium, distribution or AR rate. The possible effect of LNG as EC on sperm function remains poorly understood.
\end{abstract}

Keywords: Emergency contraception, Levonorgestrel, Acrosome reaction, Fallopian tubes, Human spermatozoa

\section{Background}

Levonorgestrel (LNG) is a progestin used in emergency contraception (EC). The currently recommended LNG dose consists of two $0.75 \mathrm{mg}$ pills taken $12 \mathrm{~h}$ apart or one dose of $1.5 \mathrm{mg}$ up to $72 \mathrm{~h}$ after unprotected sexual intercourse [1,2]. Despite the disseminated use of LNG as EC worldwide, its mechanism of action remains under debate and may involve several mechanisms that are dependent on the time of administration in relation

\footnotetext{
* Correspondence: bahamond@caism.unicamp.br

'Human Reproduction Unit, Department of Obstetrics and Gynecology, School of Medicine, University of Campinas (UNICAMP), 13084-971 Campinas, SP, Brazil

Full list of author information is available at the end of the article
}

to sexual intercourse and on the phase of the menstrual cycle in which it is taken $[3,4]$.

One of the proposed mechanisms of action concerns the effect of LNG on spermatozoa and their functions [5-8]. However, this effect is still poorly understood. Progesterone $(\mathrm{P})$ promotes changes in aspects of sperm function related to fertilization such as capacitation, sperm hyperactivation and acrosome reaction (AR) [9-15]. Although LNG exerts a weak agonistic effect on P sperm receptors, high LNG concentrations (200-800 $\mathrm{ng} / \mathrm{mL}$ ) have been shown to be capable of inducing AR in human spermatozoa in vitro [8]. However, when the spermatozoa were exposed to LNG concentrations similar to those observed in serum after intake for EC [16], no effect was observed [17]. Additionally, when human 
spermatozoa were treated in vitro with $\mathrm{LNG}$ at doses of $1 \mathrm{ng} / \mathrm{mL}, 10 \mathrm{ng} / \mathrm{mL}$ and $100 \mathrm{ng} / \mathrm{mL}$, representing lower, similar and higher levels than those observed in serum after LNG intake for EC, motility was found to be impaired with the $10 \mathrm{ng} / \mathrm{mL}$ dose; however, no effect was observed on the AR [7]. Otherwise, administration of $1.5 \mathrm{mg}$ of LNG to sterilized women at different moments following coitus failed to have any effect on the quality of cervical mucus, sperm penetration to the uterine cavity or AR [18].

After ejaculation, mammalian spermatozoa are unable to fertilize the oocyte and this capacity is subsequently acquired as a consequence of a series of physiological and functional changes called capacitation. Sperm capacitation occurs during sperm migration within the female genital tract [19]; however, it has been noted that adhesion of spermatozoa to the tubal epithelium constitutes an important condition for in vivo fertilization in several species [20]. The interaction between spermatozoa and the endosalpinx offers some protection to spermatozoa and in vitro sperm motility has been found to be longer when spermatozoa are incubated with tubal epithelium [21]. Spermatozoa undergo two forms of change in preparation for fertilization: capacitation and hyperactivation. Capacitation includes changes at the plasma membrane including the protein layer and several lipids that prepare the cells for the AR and fertilization [22]. Those spermatozoa that complete AR precociously are unable to penetrate the zona pellucida (ZP) because they lose enzymatic acrosome content [10,23].

The effect of LNG as EC on sperm function is still poorly understood and to the best of our knowledge, this effect has not been tested at the site of fertilization. Therefore, the objective of this study was to assess whether LNG, at a similar dose to those observed in serum following oral intake for EC, could affect spermatozoa exposed to human fallopian tubes under in vitro conditions.

\section{Methods}

The study was conducted at the Human Reproduction Unit, Department of Obstetrics and Gynecology, School of Medicine, University of Campinas (UNICAMP), Campinas, Brazil. All the women and semen donors gave their written informed consent and the study protocol was approved by the institution's internal review board. Women of 25-41 years of age were invited to participate in the study. The admission criteria consisted of: women who had requested surgical sterilization and for whom the abdominal route of surgery was indicated, with regular menstrual periods (25-35 day intervals), no known tubal diseases, not having used hormonal or intrauterine contraceptives during the cycle of the experiment, not having used any other form of hormone therapy, and not having been breast feeding or pregnant in the 6 months preceding the study.

\section{Experiments}

Women were instructed to use condoms as their method of contraception from the first day of the cycle until the day of surgery in order to avoid unplanned pregnancy. Follicular development was monitored daily in all participants from the $8^{\text {th }}$ day of the menstrual cycle by ultrasound with a $5.0 \mathrm{MHz}$ vaginal probe (Justavision 400, Toshiba, Toshigi-Ken, Japan). When the dominant follicle reached a diameter of 14-17 mm, surgical sterilization was scheduled for the following day. All in vitro experiments were performed prior to ovulation to avoid the effect of the P milieu on AR and any possible confounding effect of $\mathrm{P}$ on sperm adhesion to the tubal epithelium. To confirm the follicular phase of the menstrual cycle, a blood sample was drawn on the day of surgery and the serum was separated and stored at $-20^{\circ} \mathrm{C}$ until analysis. Serum P levels $<3 \mathrm{ng} / \mathrm{mL}$ were considered as confirmation of the follicular phase of the cycle [24]. P levels were measured in duplicate using a commercial electrochemiluminescence immunoassay (Roche Diagnostics GmbH, Mannheim, Germany) with a measurement range of $0.030-60.0 \mathrm{ng} / \mathrm{ml}$, an interassay coefficient of variation (CV) of $2.4 \%$ and an intraassay $\mathrm{CV}$ of $2.7 \%$.

All the women were anesthetized and mini-laparotomy was then performed. Both fallopian tubes were removed gently from the proximal portion. The side on which the dominant follicle was present was identified and recorded. The tubes were placed in two separate Petri dishes with HEPES-buffered modified Human Tubal Fluid medium (HTF-HEPES; Irvine Scientific, Santa Ana, CA, USA) and transferred immediately to the laboratory. The excess tissue (mesosalpinx) was removed.

\section{Semen samples}

Fifteen semen samples were obtained from healthy donors with normal sperm according to the analysis criteria defined in the World Health Organization manual [25]. Semen was collected by masturbation in sterile plastic jars after 3 days of sexual abstinence. After evaluation, the sample was separated into two tubes of equal volume. Motile spermatozoa of each fraction were selected by a swim-up technique in which $1 \mathrm{~mL}$ of HTF-HEPES medium supplemented with $35 \mathrm{mg} / \mathrm{mL}$ of bovine serum albumin (BSA), $1 \mathrm{nM}$ of estradiol $\left(\mathrm{E}_{2}\right)$ and $25 \mathrm{nM}$ of LNG $(0.1 \%$ solution obtained from a stock solution containing $25 \mu \mathrm{M}$ of LNG in ethanol) were used in one tube, while the same final concentration of ethanol present in the LNG-treated sample was added 
to the other tube. After $1 \mathrm{~h}$ of incubation, the supernatant with the motile spermatozoa was carefully removed and an aliquot was placed into a Makler counting chamber (Sefi Medical Instruments, Haifa, Israel), warmed to $37^{\circ} \mathrm{C}$ and examined under a microscope. After evaluation, each fraction was re-suspended in the same medium to achieve a sperm concentration of $10 \times 10^{6}$ motile spermatozoa $/ \mathrm{mL}$. A sample of each sperm suspension was taken to evaluate the AR as described below.

\section{Fallopian tube perfusion}

Using a blunt needle, one of the fallopian tubes was perfused from the proximal to the distal portion with 100 $\mu \mathrm{L}$ of a sperm suspension containing $1 \times 10^{6}$ motile spermatozoa in the medium described above, including LNG, while in the other tube the LNG in the sperm suspension was substituted for the same concentration of ethanol present in the LNG-treated sample. The fallopian tube corresponding to the ovary with the dominant follicle received the suspension containing LNG, while the other tube received the suspension containing only the vehicle. Therefore, the tube that received LNG (either the right or left tube) varied in the women according to the presence of the dominant follicle in the ipsilateral ovary. Perfusion was carried out very slowly to avoid spilling any of the solution outside the tube. The procedure was performed outside the culture medium, over a glass, under controlled temperature conditions. After perfusion with the sperm suspension, the tubes were incubated separately at $37^{\circ} \mathrm{C}$ for $4 \mathrm{~h}$ in a Petri dish containing the same medium used in the sperm suspension to allow capacitation to occur.

\section{Fallopian tube processing}

After incubation, the tubes were sectioned to separate the isthmus and the ampulla, and each segment was flushed twice, first with $5 \mathrm{ml}$ of HTF-HEPES medium and then with an equal volume of phosphate-buffered saline (PBS; GIBCO, BRL, Life Technologies, Inc, Grand island, NY, USA) medium containing 0.5\% Triton-X100 (Sigma-Aldrich, St Louis, Missouri, USA), a nonionic surfactant, in order to remove the spermatozoa adhering to the oviductal epithelium $[26,27]$. The material flushed from the tubal segments was centrifuged and the pellets were re-suspended in $100 \mu \mathrm{L}$ of PBS. The material from the first flushing with HTF-HEPES medium was evaluated to assess the number of motile sperm and the AR rate. The material obtained from flushing with TritonX100 was evaluated to asses only the number of recovered spermatozoa since all the sperm cells died when in contact with the Triton-X100 medium. The culture medium from the Petri dish in which the tubes were immersed during incubation was transferred to test tubes and centrifuged and the pellets were also diluted in $100 \mu \mathrm{L}$ of PBS for further evaluation of the number of motile sperm and AR rate.

The samples obtained following flushing with Triton were evaluated in a Neubauer chamber to verify the number of spermatozoa recovered in each segment. The other samples were divided to allow $50 \mu \mathrm{L}$ to be used for counting the number of motile spermatozoa in a Makler counting chamber and another $50 \mu \mathrm{L}$ to be used for analysis using Hoechst stain. A volume of $50 \mu \mathrm{L}$ of 1 $\mu \mathrm{g} / \mathrm{mL}$ Hoechst 33258 (bisbenzimide; Sigma Chemical Co; St. Louis USA, B2883) was added to the $50 \mu \mathrm{L}$ aliquot of the washing medium containing the recovered spermatozoa. The mixture was incubated for $5 \mathrm{~min}$ at $37^{\circ} \mathrm{C}$, washed twice with PBS to remove the excess stain and then centrifuged. The pellets were re-suspended in $50 \mu \mathrm{L}$ of PBS. Two slides from each washing were prepared and allowed to dry in a dark environment at room temperature.

\section{Assessment of AR status}

The fluorescent probe fluorescein isothiocyanate-labelled Pisum sativum agglutinin (FITC-PSA; Sigma-Aldrich) was used to evaluate AR status. After being left to dry, the slides prepared from the spermatozoa suspension stained with Hoechst were immersed in cool absolute methanol at $-20^{\circ} \mathrm{C}$ for $30 \mathrm{~s}$. Next, the slides were stained by immersing them in FITC-PSA at a concentration of $40 \mu \mathrm{g} / \mathrm{mL}$ in PBS for $30 \mathrm{~min}$, protected from light at room temperature. After incubation, the slides were washed in PBS and stored in the dark until analysis of AR and vitality. The slides were evaluated using a fluorescent microscope (Zeiss, Axioplan II, Jena, Germany) equipped with a specific filter for the FITC-PSA method (494-blue excitation; 520 emission; 510-514 barrier) and with a filter for the Hoechst 33258 stain (343-UV excitation; 480 emission; 400 barrier). Two hundred cells were evaluated in randomly selected fields.

The spermatozoa that were considered acrosomereacted were those with the following patterns: (i) patchy fluorescence in the acrosome region (partially acrosome reacted) and (ii) fluorescence of the equatorial band only (acrosome reacted) [28]. The AR rate was defined as the difference between the rate observed in the perfusion material prior to incubation and the rate observed in sperm suspension obtained after the segment was flushed.

\section{Statistical analysis}

The SAS statistical software program, version 9.2, was used to analyze the data. The mean number of recovered spermatozoa and the AR rate with and without LNG were compared using the Mann-Whitney test. The level of significance was established at $\mathrm{p}<0.05$ and all 
values are shown as means \pm standard error of the mean (SEM).

\section{Results}

Fifteen experiments were conducted. However, two women had P levels $>3 \mathrm{ng} / \mathrm{mL}$ on the day of the experiment and were excluded from the analysis to avoid the effect of P on the AR. Most of the spermatozoa recovered after the first tubal flushing was motile. The number of motile spermatozoa recovered in the isthmus was similar, irrespective of whether LNG had been added or not. Nevertheless the number of recovered motile spermatozoa was almost 10 times greater at the ampulla than at the isthmus, although there was also no difference with respect to whether LNG had been added to the medium or not. When the number of spermatozoa recovered after flushing with Triton was considered, the addition of LNG was found to have had no effect and the numbers were fairly similar, although the number of spermatozoa recovered from the ampulla was $\sim 5$ times greater than the number recovered from the isthmus (Table 1). There were no significant differences between the number of recovered motile spermatozoa in the medium to which LNG had been added and the medium to which LNG had not been added. The values found in the medium were almost five times greater than those observed when the number of motile spermatozoa at the isthmus and the number at the ampulla are taken together. Of the samples obtained after flushing with Triton, two samples had to be excluded from each segment because of technical problems that occurred during recovery of the sperm cells (Tables 1 and 2).

The number of motile spermatozoa recovered was similar both in the tubal isthmus and in the ampulla irrespective of whether LNG had been added or not. There was no difference in the number of motile spermatozoa recovered from the tubes irrespective of whether the tube was from the side with the dominant follicle or from the opposite side. In addition, there were no statistically significant differences in the number of spermatozoa recovered after flushing with Triton. Nevertheless, the number of spermatozoa was greater in the ampulla than in the isthmus on both sides irrespective of whether LNG had been added or not (Table 2).

After preparing the slides for evaluation of the AR, some were found to contain numerous epithelial cells and red and white blood cells, making it almost impossible to evaluate the AR rate accurately. For this reason, some of the evaluations were excluded from the analysis (Tables 3 and 4). The AR rate was also similar in the spermatozoa recovered from the isthmus and in those recovered from the ampulla irrespective of whether the cells had been treated or not with LNG. In addition, there were no significant differences between the AR rates in the medium irrespective of whether it had been treated with LNG or not (Table 3). No statistically significant differences were found in AR in the spermatozoa from the isthmus compared to those from the ampulla, in the medium to which LNG had been added

Table 1 Total number of recovered spermatozoa $\left(\times 10^{3}\right)$ according to the segment of the Fallopian tube and treatment

\begin{tabular}{|c|c|c|c|c|c|}
\hline & LNG in the medium & $\mathrm{N}$ & Sperm count (Mean \pm SEM) & Range & $p$-value \\
\hline \multirow[t]{3}{*}{ Isthmus* } & & & & & 0.66 \\
\hline & Yes & 13 & $4.6 \pm 1.7$ & $0-20$ & \\
\hline & No & 13 & $3.8 \pm 1.7$ & $0-15$ & \\
\hline \multirow[t]{3}{*}{ Isthmus with Triton** } & & & & & 0.23 \\
\hline & Yes & 11 & $0.5 \pm 0.2$ & $0-2$ & \\
\hline & No & 11 & $1.6 \pm 0.7$ & $0-7$ & \\
\hline \multirow[t]{3}{*}{ Ampulla* } & & & & & 0.54 \\
\hline & Yes & 13 & $38.9 \pm 11.8$ & $0-130$ & \\
\hline & No & 13 & $33.5 \pm 12.1$ & $0-140$ & \\
\hline \multirow[t]{3}{*}{ Ampulla with Triton** } & & & & & 0.71 \\
\hline & Yes & 11 & $6.8 \pm 2.8$ & $0-26$ & \\
\hline & No & 11 & $5.2 \pm 3.0$ & $0-33$ & \\
\hline \multirow[t]{3}{*}{ Medium* ${ }^{*}$} & & & & & 0.30 \\
\hline & Yes & 13 & $181.9 \pm 87.8$ & $0-1,200$ & \\
\hline & No & 13 & $217.7 \pm 85.4$ & $30-1,200$ & \\
\hline
\end{tabular}

*Motile spermatozoa recovered

**The spermatozoa from the flushing with Triton were considered adhered to the Fallopian epithelium.

Medium: refers to the medium used in the experiments in which the tubes were immersed during incubation. 
Table 2 Total number of recovered spermatozoa $\left(\times 10^{3}\right)$ according to the side with or without ovarian follicle and according to the segment of the tube and treatment

\begin{tabular}{|c|c|c|c|c|c|}
\hline & LNG in the medium & $\mathrm{N}$ & Sperm count (Mean \pm SEM) & Range & $p$-value \\
\hline \multicolumn{6}{|l|}{ With ovarian follicle } \\
\hline \multirow[t]{3}{*}{ Isthmus } & & & & & 0.26 \\
\hline & Yes & 6 & $2.5 \pm 1.7$ & $0-10$ & \\
\hline & No & 7 & $7.1 \pm 2.6$ & $0-15$ & \\
\hline \multirow[t]{3}{*}{ Isthmus with Triton* } & & & & & 0.14 \\
\hline & Yes & 5 & $0.2 \pm 0.2$ & $0-1$ & \\
\hline & No & 6 & $2.3 \pm 1.1$ & $0-7$ & \\
\hline \multirow[t]{3}{*}{ Ampulla } & & & & & 0.40 \\
\hline & Yes & 6 & $51.7 \pm 23.7$ & $0-130$ & \\
\hline & No & 7 & $22.9 \pm 10.4$ & $0-80$ & \\
\hline \multirow[t]{3}{*}{ Ampulla with Triton* } & & & & & 1.00 \\
\hline & Yes & 5 & $8.8 \pm 5.5$ & $0-26$ & \\
\hline & No & 6 & $3.8 \pm 2.0$ & $0-12$ & \\
\hline \multicolumn{6}{|l|}{ Medium } \\
\hline & Yes & 6 & $67.5 \pm 31.2$ & $0-190$ & 0.12 \\
\hline & No & 7 & $300.7 \pm 152.6$ & $30-1200$ & \\
\hline \multicolumn{6}{|c|}{ Without ovarian follicle } \\
\hline \multirow[t]{3}{*}{ Isthmus } & & & & & 0.07 \\
\hline & Yes & 7 & $6.4 \pm 2.8$ & $0-20$ & \\
\hline & No & 6 & 0.0 & 0 & \\
\hline \multirow[t]{3}{*}{ Isthmus with Triton* } & & & & & 1.00 \\
\hline & Yes & 6 & $0.7 \pm 0.3$ & $0-2$ & \\
\hline & No & 5 & $0.8 \pm 0.6$ & $0-3$ & \\
\hline \multirow[t]{3}{*}{ Ampulla } & & & & & 1.00 \\
\hline & Yes & 7 & $27.9 \pm 8.7$ & $0-65$ & \\
\hline & No & 6 & $45.8 \pm 23.6$ & $0-140$ & \\
\hline \multirow[t]{3}{*}{ Ampulla with Triton* } & & & & & 0.52 \\
\hline & Yes & 6 & $5.2 \pm 2.8$ & $0-18$ & \\
\hline & No & 5 & $6.8 \pm 6.6$ & $0-33$ & \\
\hline \multirow[t]{3}{*}{ Medium } & & & & & 0.68 \\
\hline & Yes & 7 & $280.0 \pm 156.4$ & $30-1200$ & \\
\hline & No & 6 & $120.8 \pm 43.3$ & $30-285$ & \\
\hline
\end{tabular}

*The spermatozoa from the flushing with Triton were considered adhered to the Fallopian epithelium.

Medium: refers to the medium used in the experiments in which the tubes were immersed during incubation.

Table 3 Percentage of spermatozoa with acrosomal reaction according to the segment of the Fallopian tube and treatment

\begin{tabular}{|c|c|c|c|c|}
\hline & LNG in the medium & $\mathrm{N}$ & Acrosome reacted spermatozoa (\%) (Mean \pm SEM) & $p$-value \\
\hline Isthmus & & & & 0.57 \\
\hline & Yes & 9 & $15.2 \pm 3.3$ & \\
\hline & No & 9 & $18.7 \pm 2.5$ & \\
\hline Ampulla & & & & 0.96 \\
\hline & Yes & 9 & $9.1 \pm 1.2$ & \\
\hline & No & 8 & $9.4 \pm 1.9$ & \\
\hline Medium & & & & 0.28 \\
\hline & Yes & 9 & $5.5 \pm 1.9$ & \\
\hline & No & 9 & $3.8 \pm 2.1$ & \\
\hline
\end{tabular}


compared to the medium without LNG, or on the side of the dominant ovarian follicle compared to the opposite side. Furthermore, no statistically significant differences were found in the percentage of AR observed in the spermatozoa from the tubal segments and medium as a function of whether they were obtained from the side with the dominant ovarian follicle or not (Table 4).

\section{Discussion}

These results show that the addition of LNG did not significantly affect the number of recovered motile spermatozoa either at the isthmus or at the ampulla nor did it affect the number of recovered spermatozoa adhered to the tubal epithelium. With respect to whether the addition of LNG affected the number of recovered motile spermatozoa, the number of spermatozoa adhered to the tubal epithelium or the AR rate in the flushing medium on the side with the dominant ovarian follicle or on the opposite side, no statistically significant differences were found.

However, the principal limitation of this study refers to the design and development of the procedures. The experiments were conducted in vitro and, although the spermatozoa were tested in the tubal environment, conditions differed from those encountered in vivo. The doses of LNG used for flushing the tubes are similar to serum levels found after oral intake as EC and possibly higher than those found in oviductal fluid. In fact, as previously reported by our group [18], the LNG concentration in uterine flushing after oral intake was less than $2 \%$ of that found in serum [16] and concentrations at the tubal lumen are probably similar. Furthermore, these findings do not permit us to affirm that the administration of LNG as EC in vivo affects the oviductal microenvironment, impairing sperm function. In fact, the best experiment may be for women to take a dose of LNG as EC prior to having their tubes removed to evaluate whether or not LNG exerts any effect on oviductal $P$ receptors and whether it is able to modify the oviductal microenvironment and its interaction with spermatozoa. The experiments conducted in the present study did not mimic this situation.

Previous in vitro experiments have shown that at a dose of $10 \mathrm{ng} / \mathrm{mL}$, LNG was unable to induce AR $[7,17]$ and also failed to induce any significant changes in the number or AR status of spermatozoa recovered from the uterus after administration of $1.5 \mathrm{mg}$ of LNG to sterilized women at different times following coitus [18]. However, to the best of our knowledge, no studies have been conducted in which the effect of LNG on spermatozoa was evaluated at the site of fertilization. With the intention of contributing to the understanding of the

Table 4 Percentage of spermatozoa with acrosomal reaction according to the side with or without ovarian follicle and according to the segment of the fallopian tube and treatment

\begin{tabular}{|c|c|c|c|c|}
\hline & LNG in the medium & $\mathbf{N}$ & Acrosome reacted spermatozoa $(\%)($ Mean \pm SEM) & $p$-value \\
\hline \multicolumn{5}{|c|}{ With ovarian follicle } \\
\hline \multirow[t]{3}{*}{ Isthmus } & & & & 0.15 \\
\hline & Yes & 4 & $7.9 \pm 3.2$ & \\
\hline & No & 5 & $19.9 \pm 3.9$ & \\
\hline \multirow[t]{3}{*}{ Ampulla } & & & & 0.35 \\
\hline & Yes & 4 & $7.5 \pm 1.9$ & \\
\hline & No & 5 & $11.2 \pm 2.5$ & \\
\hline \multirow[t]{3}{*}{ Medium } & & & & 0.77 \\
\hline & Yes & 3 & $5.5 \pm 3.1$ & \\
\hline & No & 5 & $2.8 \pm 3.8$ & \\
\hline \multicolumn{5}{|c|}{ Without ovarian follicle } \\
\hline \multirow[t]{3}{*}{ Isthmus } & & & & 0.42 \\
\hline & Yes & 5 & $21.0 \pm 3.8$ & \\
\hline & No & 4 & $17.1 \pm 3.4$ & \\
\hline \multirow[t]{3}{*}{ Ampulla } & & & & 0.40 \\
\hline & Yes & 5 & $10.4 \pm 1.5$ & \\
\hline & No & 3 & $6.3 \pm 2.6$ & \\
\hline \multirow[t]{3}{*}{ Medium } & & & & 0.54 \\
\hline & Yes & 6 & $5.5 \pm 2.6$ & \\
\hline & No & 4 & $5.1 \pm 1.5$ & \\
\hline
\end{tabular}

Medium: refers to the medium used in the experiments in which the tubes were immersed during incubation. 
mechanism of action involved in the contraceptive effect of LNG as an EC, the objective of the present study was to determine whether the drug was able to affect the sperm count, the number of spermatozoa adhered to the tubal epithelium, their distribution and $A R$ in human fallopian tubes in vitro.

The methodology used in this study could not be better. Initially, an attempt was made to recover spermatozoa from tubes obtained at the time of sterilization from women who had had unprotected coitus and received LNG as EC. However, the number of spermatozoa recovered after perfusion of the tubes was extremely low and did not permit any further analysis to be made, a result that had also been reported previously using the same technique [29]. Consequently, it was decided to work in vitro, perfusing the tubes with a predetermined number of spermatozoa and evaluating sperm function after incubation.

It is well known that women are fertile for six days in the menstrual cycle: the five days preceding ovulation and the day of ovulation itself [30]. Recently Noé et al. [31] observed that despite the evidence of follicular rupture in women who had received LNG as EC prior to follicular rupture, no pregnancies occurred among those women, suggesting that mechanisms other than ovulation suppression prevent pregnancy when LNG is administered as EC to exposed women.

Sperm migration to the oviducts and adhesion to the tubal epithelium, a well-known mechanism of achieving fertilization, are controlled by sex steroids. In rats, $E_{2}$ has been reported to facilitate sperm migration from the uterus to the oviducts and P modulated this effect [27]. In addition, the interaction between $\mathrm{E}_{2}$ and $\mathrm{P}$ stimulated sperm adhesion to the tubal epithelium. Although no different sperm reservoir has been found in human tubes, a functional reservoir has been described and in vitro, spermatozoa were observed adhered to the epithelium of the isthmus, albeit in an intermittent manner [32,33]. Ortiz et al. [34] observed in vitro that after $3 \mathrm{~h}$ of incubation, the number of spermatozoa adhered per $0.1 \mathrm{~mm}^{2}$ of tubal explants decreased both at the isthmus and at the ampulla when LNG was added. However, in the present study, a different methodology was used in that the tubal segments were incubated for $4 \mathrm{~h}$ and flushed with a nonionic surfactant (Triton-X100). In addition, the number of spermatozoa obtained in each segment of the tube was recorded separately. LNG had no effect on the number of adhered spermatozoa recovered or on their distribution along the fallopian tubes.

When ovulation is about to take place, spermatozoa undergo capacitation and hyperactivation and are able to progress to the tubal ampulla. In addition, $\mathrm{P}$ modulates many aspects of sperm function and mimics the AR-inducing properties of the follicular fluid almost perfectly, while the effects of $\mathrm{P}$ on sperm function are mediated by receptors located in the plasma membrane, [35] defined as nongenomic, since they are rapid and do not involve transcriptional processes [36]. It is well known that response is absolutely dependent on the presence of extracellular bicarbonate, $[37,38]$ which is present in intraluminal oviductal fluid at levels higher than those found in peripheral blood [39].

There are two classes of nongenomic $P$ receptors in the human spermatozoa. One has an elevated affinity (at nanomolar concentrations) and is specific for $\mathrm{P}$, while the other has low affinity (micromolar) and also binds to other hydroxylated P derivatives [36]. When P activates human spermatozoa, an increase is seen in intracellular free calcium that may trigger the process of AR [36,40]. Progestogens such as LNG are unable to mimic the effect of $\mathrm{P}$ in elevating intracellular calcium and appear to act as very weak agonists in nongenomic receptors compared to their potent effect on genomic receptors [40].

With respect to the effect of $\mathrm{P}$ on the AR process, some studies were carried out to evaluate whether LNG was able to affect sperm function as P does. A direct relationship was found between an increase in the AR rate and higher LNG concentrations, suggesting that at higher LNG concentrations (200-800 ng/mL) the nongenomic P receptors on the surface of spermatozoa are able to recognize the progestogen molecule and exert their effect [8]. Nevertheless, the studies that used a similar dose of LNG to that observed in serum following oral intake for EC failed to show any effect of LNG on AR in spermatozoa in vitro $[7,17]$ or in spermatozoa recovered from the uterus [18].

\section{Conclusions}

In conclusion, the hypothesis that LNG, in a similar dose to those found in the serum of women following oral intake for EC, may affect the number of motile spermatozoa, the adhesion of sperm to the tubal epithelium, distribution and the AR of spermatozoa in the fallopian tubes in vitro was not confirmed in the present study. The present findings also failed to show any effect on motile spermatozoa recovered after exposure to the fallopian tubes despite the fact that the doses of LNG used in vitro are probably higher than those reached within the oviduct in vivo.

\section{Abbreviations}

AR: Acrosome reaction; CV: Coefficient of variation; EC: Emergency contraception; $E_{2}$ : Estradiol; FITC-PSA: Fluorescein isothiocyanate-labelled Pisum sativum agglutinin; HTF-HEPES: HEPES-buffered modified human tubal fluid medium; LNG: Levonorgestrel; P: Progesterone; SEM: Standard error of the mean; ZP: Zona pellucida

\section{Acknowledgements}

A.H was a fellow at the Fundação de Amparo a Pesquisa do Estado de São Paulo 
(FAPESP) and partial financial support was obtained from this institution under grant \#2007/07866-2 and also from the Brazilian Conselho Nacional de Pesquisa e Desenvolvimento Científico e Tecnológico (CNPq) under grant \#573747/2008-3.

\section{Author details}

Human Reproduction Unit, Department of Obstetrics and Gynecology, School of Medicine, University of Campinas (UNICAMP), 13084-971 Campinas, SP, Brazil. ${ }^{2}$ Instituto Chileno de Medicina Reproductiva (ICMER), Santiago, Chile. ${ }^{3}$ Departamento de Biología, Facultad de Química y Biología, Universidad de Santiago de Chile, Santiago, Chile.

\section{Authors' contributions}

$A H, M E O, H B C$ and $L B$ participated in the design of the study and in developing the research protocol; AH, MVB, FF, NMM, MHRRG, and LB conducted the study at the outpatient clinic, surgical theatre and at the laboratory. All the authors contributed equally in writing the manuscript and in reviewing and revising it. All authors read and approved the final manuscript.

\section{Competing interests}

The authors declare that they have no competing interests.

Received: 6 September 2011 Accepted: 30 January 2012

Published: 30 January 2012

\section{References}

1. Tremblay D, Gainer E, Ulmann A: The pharmacokinetics of $750 \mu \mathrm{g}$ levonorgestrel following administration of one single dose or two doses at 12- or 24 interval. Contraception 2001, 64:327-331.

2. Von-Hertzen H, Piaggio G, Ding J, Chen J, Song S, Bártfai G, Ng E, GemzellDanielsson K, Oyunbileg A, Wu S, Cheng W, Lüdicke F, Pretnar-Darovec A, Kirkman R, Mittal S, Khomassuridze A, Apter D, Peregoudov A, WHO Research Group on Post-ovulatory Methods of Fertility Regulation: Low doses of mifepristone and two regimens of levonorgestrel for emergency contraception: a WHO randomised trial. Lancet 2002, 360:1803-1810.

3. Croxatto HB, Devoto L, Durand M, Ezcurra E, Larrea F, Nagle C, Ortiz ME, Vantman D, Vega M, Von-Hertzen H: Mechanism of action of hormonal preparations used for emergency contraception: a review of the literature. Contraception 2001, 63:111-121.

4. Durand $\mathrm{M}$, Cravioto CM, Raymond EG, Raymond EG, Durán-Sánchez $\mathrm{O}$, De la-Luz Cruz-Hinojosa M, Castell-Rodríguez A, Schiavon R, Larrea F: On the mechanism of action of short-term levonorgestrel administration in emergency contraception. Contraception 2001, 64:227-234.

5. Kesseru E, Garmendia F, Westphal N, Parada J: The hormonal and peripheral effects of dl-norgestrel in postcoital contraception. Contraception 1974, 10:411-424

6. Nikkanen V, Söderström K, Tuusa S, Jaakkola UM: Effect of local epididymal levonorgestrel on the fertilizing ability of male rat, a model for posttesticular contraception. Contraception 2000, 61:401-406.

7. Yeung WSB, Chiu PCN, Wang CH, Yao YQ, Ho PC: The effects of levonorgestrel on various sperm functions. Contraception 2002, 66:453-457.

8. Bahamondes L, Nascimento JA, Munuce MJ, Fazano F, Faundes A: The in vitro effect of levonorgestrel on the acrosome reaction of human spermatozoa from fertile men. Contraception 2003, 68:55-59.

9. Osman RA, Andria ML, Jones AD, Meizel S: Steroid induced exocytosis: the human sperm acrosome reaction. Biochem Biophys Res Commun 1989, 160:828-833.

10. Blackmore B, Beebe S, Danford D, Alexander N: Progesterone and 17-alpha hydroxyprogesterone: novel stimulators of calcium influx in human sperm. J Biol Chem 1990, 265:1376-1380.

11. Sueldo CE, Oehninger S, Subias E, Mahony M, Alexander NJ, Burkman LJ, Acosta AA: Effect of progesterone on human zona pellucida sperm binding and oocyte penetrating capacity. Fertil Steril 1993, 60:136-140.

12. Oehninger S, Sueldo C, Lanzendorf S, Mahony M, Burkman L, Alexander NJ, Hodgen GD: A sequential analysis of the effect of progesterone of specific sperm functions crucial to fertilization in vitro in infertile patients. Hum Reprod 1994, 9:1322-1327.
13. Bray C, Brown JCK, Publicover S, Barratt CLR: Progesterone interaction with sperm plasma membrane, calcium influx and induction of the acrosome reaction. Reprod Med Rev 1999, 7:81-93.

14. Baldi E, Luconi M, Bonaccorsi L, Forti G: Nongenomic effects of progesterone on spermatozoa: mechanisms of signal transduction and clinical implications. Front in Biosc 1998, 3:1051-1059.

15. Baldi E, Luconi M, Bonaccorsi L, Muratori M, Forti G: Intracellular events and signaling pathways involved in sperm acquisition of fertilizing capacity and acrosome reaction. Front in Biosc 2000, 5:110-123.

16. Johansson E, Brache V, Alvarez F, Faundes A, Cochon L, Ranta S, Lovern M, Kumar N: Pharmacokinetic study of different dosing regimens of levonorgestrel for emergency contraception in healthy women. Hum Reprod 2002, 17:1472-1476.

17. Brito KS, Bahamondes L, Nascimento JA, De-Santis L, Munuce MJ: The in vitro effect of emergency contraception doses of levonorgestrel on the acrosome reaction of human spermatozoa. Contraception 2005, 72:225-228.

18. Do Nascimento JA, Seppala M, Perdigão A, Espejo-Arce X, Munuce MJ Hautala $L$, Koistinen $R$, Andrade $L$, Bahamondes $L$ : In viv assessment of the human sperm acrosome reaction and the expression of glycodelin- $A$ in human endometrium after levonorgestrel-emergency contraceptive pill administration. Human Reprod 2007, 22:2190-2195.

19. Yanagimachi R: Mammalian fertilization. In The physiology of reproduction. Edited by: Knobil E, Neill JD. New York: Raven; 1988:135-185.

20. Croxatto HB: Physiology of gamete and embryo transport through the Fallopian tube. Reprod Biomed Online 2002, 4:160-169.

21. Kervancioglu ME, Saridogan E, Aitken J, Djahanbakhch O: Importance of sperm-to-epithelial cell contact for the capacitation of human spermatozoa in fallopian tube epithelial cell cocultures. Fertil Steril 2000, 74:780-784.

22. De-Jonge C: Biological basis for human capacitation. Hum Reprod Update 2005, 11:205-214.

23. Flesch FM, Gadella BM: Dynamics of the mammalian sperm plasma membrane in the process of fertilization. Biochim et Biophys Act 2000, 1469:197-235.

24. Israel R, Mishell DR Jr, Stone SC, Thorneycroft IH, Moyer DL: Single luteal phase serum progesterone assay as an indicator of ovulation. Am J Obstet Gynecol 1972, 112:1043-1046.

25. World Health Organization: WHO laboratory manual for examination of human semen and semen-cervical mucus interaction Cambridge (UK): Cambridge, University Press; 2010.

26. Smith $\Pi$, Yanagimachi R: The viability of hamster spermatozoa stored in the isthmus of the oviduct: the importance of sperm-epithelium contact form sperm survival. Biol Reprod 1990, 42:450-457.

27. Orihuela PA, Ortíz ME, Croxatto HB: Sperm migration into and through the oviduct following artificial insemination at different stages of the estrous cycle in the rat. Biol Reprod 1999, 60:908-913.

28. Cross NL, Morales P, Overstreet JW, Hanson FW: Induction of acrosome reactions by the human zona pellucida. Biol Reprod 1988, 38:235-244.

29. Williams M, Hill CJ, Scudamore I, Dunphy B, Cooke ID, Barratt CLR: Sperm numbers and distribution within the human Fallopian tube around ovulation. Human Reprod 1993, 8:2019-2026.

30. Wilcox AJ, Weinberg CR, Baird DD: Timing of sexual intercourse in relation to ovulation. effects on the probability of conception, survival of the pregnancy, and sex of the baby. N Engl J Med 1995, 333:1517-1521.

31. Noé G, Croxatto HB, Salvatierra AM, Reyes V, Villarroel C, Muñoz C Morales $G$, Retamales A: Contraceptive efficacy of emergency contraception with levonorgestrel given before or after ovulation. Contraception 2010, 81:414-420.

32. Pacey AA, Hill CJ, Scudamore IW, Warren MA, Barratt CLR, Cooke ID: The interaction in vitro of human spermatozoa with epithelial cells from the human uterine (fallopian) tube. Hum Reprod 1995, 10:360-366.

33. Suarez SS, Pacey AA: Sperm transport in the female reproductive tract. Hum Reprod Update 2006, 12:23-37.

34. Ortiz ME, Carreño DV, Llados C, et al: Levonorgestrel interfiere con la adhesión de los espermatozoides al tejido oviductal in vitro. Proceedings of the XXI Reunión Bienal Associación Latinoamericana de Investigadores en Reproducción Humana (ALIRH); 2009, 43.

35. Calogero AE, Burrello N, Barone N, Palermo I, Grasso-D'Agata R: Effects of progesterone on sperm function: mechanisms of action. Hum Reprod 2000, 15:28-45. 
36. Luconi M, Bonaccorsi L, Maggi M, Pecchioli P, Krausz C, Forti G, Baldi E: Identification and characterization of functional nongenomic progesterone receptors on human sperm membrane. J Clin Endocrinol Metab 1998, 83:877-885.

37. Aitken RJ, Harkiss D, Knox W, Paterson M, Irvine S: On the cellular mechanisms by which the bicarbonate ion mediates the extragenomic action of progesterone on human spermatozoa. Biol Reprod 1998, 58:186-196.

38. Sauber K, Meizel S: Importance of bicarbonate to the progesteroneinitiated human sperm acrosome reaction. J Androl 1995, 16:226-271.

39. Rodriguez-Martinez H: Role of the oviduct in sperm capacitation. Theriogenology 2007, 68(1):138-146.

40. Blackmore PF, Neulin J, Lattanzio F, Beede SJ: Cell surface binding sites for progesterone mediate calcium uptake in human sperm. J Biol Chem 1991, 266:18655-18659.

doi:10.1186/1477-7827-10-8

Cite this article as: Hermanny et al:. In vitro assessment of some sperm function following exposure to levonorgestrel in human fallopian tubes. Reproductive Biology and Endocrinology 2012 10:8.

\section{Submit your next manuscript to BioMed Central} and take full advantage of:

- Convenient online submission

- Thorough peer review

- No space constraints or color figure charges

- Immediate publication on acceptance

- Inclusion in PubMed, CAS, Scopus and Google Scholar

- Research which is freely available for redistribution

Submit your manuscript at www.biomedcentral.com/submit 